\title{
A qualidade da assistência de enfermagem em uma unidade de emergência: a percepção do usuário
}

\author{
The quality of nursing care at an emergency unit: the patient's perception
}

Calidad de atención de enfermería en unidad de emergencias: percepción del paciente

Alexandre Souza Morais ${ }^{1}$, Marta Maria Melleiro²

\footnotetext{
* Extraído da dissertação de Mestrado intitulada "A qualidade da assistência de enfermagem em uma unidade de emergência de adultos: a percepção do usuário", apresentada à Escola de Enfermagem da Universidade de São Paulo em 2009.

1 Enfermeiro, Mestre em Enfermagem. Vitória, ES, Brasil. E-mail: alemorais@usp.br.

2 Enfermeira, Doutora em Enfermagem. Professora Associada da Escola de Enfermagem da Universidade de São Paulo. São Paulo, SP, Brasil. E-mail: melleiro@usp.br.
}

\section{RESUMO}

Estudo exploratório-descritivo, realizado em um hospital público e de ensino, cujo objetivo foi analisar a qualidade da assistência de enfermagem em uma unidade de emergência a partir da percepção dos usuários. A população foi constituída por 260 participantes e os dados foram coletados entre agosto e outubro de 2008 por meio de um questionário empregandose a escala Likert. Verificamos que $52,7 \%$ dos participantes pertenciam ao sexo masculino, com média de idade de 46,9 anos e a maior parte possuía ensino fundamental (54,6\%). O instrumento de coleta de dados teve Alpha de Cronbach igual a 0,88. Na comparação das dimensões de estrutura, processo e resultado, a que obteve maior escore foi a de resultado 36,20 e a que teve menor, a de estrutura com 33,20. O estudo permitiu analisar a assistência de enfermagem na referida unidade de emergência e, logo, contribuirá para a reformulação dos aspectos avaliados nas três dimensões investigadas.

Descritores: Avaliação em Saúde; Qualidade da Assistência à Saúde; Enfermagem; Enfermagem em Emergência.

\section{ABSTRACT}

The objective of this exploratory-descriptive study was to analyze the nursing care at an emergency unit based on the patients' perception. The study was performed at a public teaching hospital. The study population consisted of 260 subjects, and data collection was performed between August and October of 2008 by means of a questionnaire using a Likert scale. We found that $52.7 \%$ of the participants were male, with an average age or 46.9 years, and most had a complete primary education (54.6\%). The data collection instrument had a Cronbach's Alpha of 0.88. By comparing structure, process and outcome, the dimension with the highest score was outcome, with 36.20, while structure was the lowest, with 33.20. in conclusion, the study permitted to analyze the nursing care at the referred emergency unit, and, thus, will contribute with the reformulation of the aspects validated in the three studied dimensions.

Descriptors: Health Evaluation; Quality of Health Care; Nursing; Emergency Nursing.

\section{RESUMEN}

Estudio exploratorio-descriptivo, objetivando analizar la calidad de atención de enfermería en unidad de emergencias en la percepción de los pacientes. Investigación realizada en hospital público de enseñanza. La población se constituyó con 260 participantes, datos recolectados de agosto a octubre de 2008, mediante un cuestionario empleando la escala de Likert. Verificamos que $51,7 \%$ de los participantes eran de sexo masculino, media etaria de 46,9 años, la mayoría poseía enseñanza primaria (54,6\%). El instrumento de recolección de datos tuvo Alpha de Cronbach igual a 0,88. En la

comparación de dimensiones de estructura, proceso y resultado; la que obtuvo mayor puntaje fue la de resultado, con 36,20; y el menor fue el de estructura, con 33,20. El estudio permitió así analizar la atención de enfermería en la referida unidad de emergencias y contribuirá a la reformulación de los aspectos evaluados en las tres dimensiones estudiadas.

Descriptores: Evaluación en Salud; Calidad de la Atención de Salud; Enfermería; Enfermería de Urgencia. 


\section{INTRODUÇÃO}

As Unidades de Emergência (UE) estão inseridas no sistema de saúde brasileiro como um local onde os usuários procuram solução para as suas necessidades de saúde, sejam elas emergenciais ou não. Nessa perspectiva, observa-se que a procura por essas unidades, por vezes, ocorre em detrimento das unidades de atenção básica e está relacionada às distorções do sistema de saúde vigente.

Em decorrência desse contexto, as UE têm atendido além de sua capacidade instalada, o que representa um problema grave e relevante do sistema de saúde, principalmente, nos países em desenvolvimento. 0 agravamento progressivo desse quadro amplifica as discussões sobre os fatores relacionados com a situação caótica do atendimento de emergência em nosso país.

Os resultados da pesquisa Saúde na Opinião dos Brasileiros, realizada em 2002, por solicitação do Conselho Nacional de Secretários de Saúde (CONASS), revelam as principais causas de descontentamento da população com os serviços de saúde, entre elas aquelas relacionadas aos serviços de emergência, tais como a baixa capacidade instalada, a falta de acolhimento, a sobrecarga com atendimentos de baixa complexidade, a retaguarda inadequada e a formação dos profissionais inadequada ${ }^{(1)}$.

Cabe ressaltar que a percepção do usuário acerca dos fatores intervenientes na qualidade do atendimento vai ao encontro das causas acima apontadas. Sob essa ótica, acredita-se que pesquisas que apontem para questões referentes à satisfação dos usuários com os serviços de saúde devem ser utilizadas como parâmetros de avaliação.

A satisfação é definida como o produto da gestão dos relacionamentos e das expectativas de atendimento, referindo-se não só à qualidade do serviço, mas também ao acesso, local, cortesia, presteza da resposta e atenção humana ao usuário(2).

Pode ser compreendida, também, como a comparação das expectativas do usuário com suas percepções a respeito do encontro com o serviço real. Dessa maneira, a satisfação geral com o serviço é resultante das várias percepções que o cliente vai tendo ao longo da sequência de momentos de contato com um provedor, e que essa, portanto, ocorre na fase de consumo e na fase de pós-consumo ${ }^{(3)}$.
Diante disso, acreditamos que a satisfação do usuário é uma maneira de se avaliar a qualidade do serviço e do atendimento, cujas informações podem servir para analisar a estrutura oferecida, os processos executados e os resultados alcançados( ${ }^{(4)}$.

Nesta pesquisa entende-se, portanto, por satisfação, a percepção e a avaliação que o usuário tem sobre determinadas dimensões dos serviços de saúde.

o principal vínculo entre o usuário e o estabelecimento de saúde ocorre através do serviço de enfermagem, por se constituir no maior grupo de profissionais da saúde e que mantém um contato ininterrupto com o usuário, promovendo a manutenção, a recuperação e a reabilitação da saúde, por meio do cuidado. Isso pode influenciar no julgamento dos usuários no que tange à imagem do hospital.

A qualidade da atenção à saúde é difícil de ser definida e envolve alguns critérios, determinados em forma de juízos de valor, que podem ser aplicados a distintos aspectos, propriedades, componentes ou alcances desse tipo de atenção(5).

Ao considerar o usuário como receptáculo do cuidado, a qualidade poderá ser considerada uma construção social, produzida a partir de referências dos sujeitos envolvidos, que atribuem significados às suas experiências, privilegiando ou excluindo determinados aspectos segundo uma hierarquia de preferências( ${ }^{(6)}$.

A qualidade da assistência à saúde é definida como a obtenção de maiores benefícios em detrimento de menores riscos para o usuário, benefícios estes que, por sua vez, definem-se em função do alcançável de acordo com os recursos disponíveis e os valores sociais existentes. A qualidade da assistência não é um atributo abstrato e, sim, que é construída pela avaliação assistencial abrangendo a análise da estrutura, dos processos de trabalho e dos resultados dos mesmos, estabelecendo um modelo avaliativo em saúde pautado em componentes de estrutura, de processo e de resultado ${ }^{(7-8)}$.

O modelo avaliativo Donabediano constituído pela tríade de estrutura, processo e resultado, propõe-se a avaliar os serviços de saúde mediante as seguintes dimensões:

- Estrutura: corresponde às características relativamente estáveis da instituição, tais como 
estrutura organizacional, recursos humanos, físicos, materiais e financeiros;

- Processo: refere-se aos procedimentos empregados e ao desenvolvimento das atividades dos profissionais envolvidos na prestação de serviços ao usuário, bem como a relação profissional-usuário;

- Resultado: caracteriza-se pela consequência das atividades realizadas, sendo a demonstração da combinação de fatores do meio ambiente, da estrutura e do processo.

Considerando a realidade que envolve as UE e o referencial de qualidade em saúde exposto, bem como a lacuna existente na literatura sobre avaliação de serviços de emergência na perspectiva de usuários, acreditamos que este estudo trará subsídios para a reorganização do referido serviço, no que tange aos aspectos de estrutura, processo e resultado.

Frente ao exposto, o objetivo deste estudo foi analisar a qualidade da assistência de enfermagem, em uma Unidade de Emergência de Adultos, de um hospital de ensino, na percepção dos usuários.

\section{MÉTODO}

Trata-se de um estudo exploratório-descritivo, de abordagem quantitativa, realizado em um hospital público e de ensino, localizado em um Município do interior do Estado de São Paulo, cuja unidade de emergência atende a uma média de 500 usuários/dia. Este hospital é considerado referência terciária, sendo a principal porta de entrada para urgências e emergências, bem como retaguarda de serviços de diagnóstico para a região metropolitana de sua área de abrangência.

A amostra foi constituída pelos usuários atendidos na Unidade Emergência Adulto (UEA), conforme os seguintes critérios de elegibilidade: idade igual ou superior a 18 anos e alfabetizados; estarem na unidade de emergência por, no mínimo, seis horas e apresentarem condições clínicas favoráveis ao responder o instrumento de coleta de dados. Após o cálculo estatístico, a amostra foi estabelecida em 260 sujeitos.

O instrumento para coleta de dados foi constituído de duas partes: a primeira contendo os dados sociodemográficos dos participantes e a segunda com 33 proposições referentes às dimensões de estrutura (recursos humanos, materiais e físicos), processo (atividades desenvolvidas e relacionamento com os usuários) e resultado de serviços de emergência (resposta ao atendimento prestado), sendo 20 proposições de cunho positivo e 13 proposições negativas, empregandose uma escala psicométrica (Escala de Likert) com cinco graus, a saber: concordo totalmente, concordo parcialmente, indiferente, discordo parcialmente e discordo totalmente.

O instrumento foi submetido à validação de conteúdo por sete especialistas nas temáticas envolvidas, sendo aprimorado a partir dos comentários e sugestões dos mesmos. A seguir, a confiabilidade de coerência interna do instrumento foi avaliada, sendo calculada por meio do teste de Alpha de Cronbach; o valor alcançado foi igual a 0,88 , indicando que foi possível medir $88 \%$ do processo real.

O estudo foi aprovado pelo Comitê de Ética em Pesquisa da referida instituição, sob o protocolo $n^{\circ}$ 061914/2005-91 e a coleta de dados ocorreu nos meses de agosto a outubro 2008. O nome da instituição, cenário deste estudo, foi omitido, devido ao compromisso de anonimato assumido. Os usuários que atenderam aos critérios de elegibilidade foram abordados a respeito dos objetivos da pesquisa e os que concordaram em participar assinaram o Termo de Consentimento Livre e Esclarecido.

Os dados foram armazenados em planilha eletrônica Excel ${ }^{\circledR}$ e analisados por meio do processamento dos recursos de computação do sistema Statistic Package for Social Sciences (SPSS).

\section{RESULTADOS}

A amostra que compôs este estudo caracterizou-se por participantes com idade entre 18 e 92 anos, sendo a média de 46,99 anos ( $d p \pm 18,12)$ e a mediana de 44 anos, com predomínio do sexo masculino - 52,7\%. Grande parte dos participantes, 173 (66,5\%), não possuía o ensino médio completo e apenas uma pequena parcela havia completado o ensino superior $3(1,2 \%)$.

No sentido de comparar os escores pontuados pelos usuários, foram estabelecidas as variáveis relacionadas à caracterização do atendimento, descritas na Tabela 1. 
Tabela 1: Distribuição dos usuários segundo a caracterização dos atendimentos na UEA. Município da Região Metropolitana de Campinas, SP, 2008.

\begin{tabular}{ccc}
\hline Variável & & Valores \\
\hline & & $127(48,8 \%)$ \\
Tempo de permanência (em horas) & $24(9,2 \%)$ & $109(41,9 \%)$ \\
& 12 l--- 24 & $(23,1 \%)$ \\
Motivo da internação & $\geq 24$ & $108(41,5 \%)$ \\
& Não urgência & $75(28,8 \%)$ \\
& Semi urgência & $17(6,5 \%)$ \\
& Urgência & 0 (0,0\%) \\
& Emergência & $129(49,6 \%)$ \\
Setor & Ressuscitação & $39(15,0 \%)$ \\
& Azul & $41(15,8 \%)$ \\
\end{tabular}

No que tange ao tempo de permanência, observa-se, pela Tabela 1, a predominância de usuários nos extremos: o período de seis a 12 horas composto por 127 (48,8\%) usuários e $\geq 24$ horas por 109 (41,9\%).

A percepção dos usuários acerca da unidade de emergência de adultos foi categorizada nas dimensões de estrutura, processo e resultado do modelo avaliativo Donabediano, as quais se encontram descritas a seguir.

\section{Dimensão de estrutura}

$\mathrm{Na}$ dimensão de estrutura foram avaliadas as questões que implicavam nas características relativamente estáveis da instituição como recursos humanos, materiais, físicos, financeiros e o modelo organizacional.

A escolha dos 11 elementos a serem avaliados obedeceu à lógica da finalidade da UEA, sendo agrupados em recursos físicos, materiais e humanos.

Tabela 2: Percentual das respostas referentes às proposições para avaliação da dimensão estrutura. Município da Região Metropolitana de Campinas, SP, 2008.

\begin{tabular}{|c|c|c|c|c|c|c|}
\hline \multirow{2}{*}{$\mathbf{R}$} & \multirow{2}{*}{ Proposições } & \multicolumn{5}{|c|}{ Escala de Likert } \\
\hline & & 1 & 2 & 3 & 4 & 5 \\
\hline \multirow{6}{*}{ Físıcos } & Foi fácil chegar a unidade de emergência & 5 & 0,8 & 7,7 & 11,5 & 75 \\
\hline & O estado de conservação da unidade era precário. & 36,9 & 18,5 & 9,6 & 28,1 & 6,9 \\
\hline & O local onde fui atendido era confortável. & 6,2 & 5,4 & 7,7 & 35,4 & 45,4 \\
\hline & Os banheiros não eram limpos. & 9,2 & 5 & 13,5 & 33,8 & 38,5 \\
\hline & O local onde fiquei não permitiu minha privacidade & 43,1 & 7,3 & 15 & 18,8 & 15,8 \\
\hline & $\begin{array}{l}\text { Percebi que havia placas de sinalização, indicando os setores da } \\
\text { unidade de emergência. }\end{array}$ & 5 & 1,2 & 8,5 & 25,8 & 59,6 \\
\hline \multirow{3}{*}{ MATERIAIS } & Faltaram roupas para meu atendimento. & 48,8 & 3,5 & 38,1 & 3,8 & 5,8 \\
\hline & Faltaram equipamentos para o meu atendimento. & 80 & 2,3 & 6,2 & 5,4 & 6,2 \\
\hline & A unidade dispunha de todos os medicamentos que necessitei. & 3,5 & 2,7 & 4,2 & 10,8 & 78,8 \\
\hline \multirow{2}{*}{ HUMANOS } & Os profissionais de enfermagem eram capacitados. & 0,8 & 0 & 5,4 & 14,6 & 79,2 \\
\hline & Faltaram profissionais de enfermagem para meu atendimento. & 77,7 & 4,2 & 3,1 & 9,6 & 5,4 \\
\hline
\end{tabular}

\section{Dimensão Processo}

A dimensão de processo refere-se ao conjunto de atividades desenvolvidas na produção em geral, e no setor saúde, nas relações estabelecidas entre os profissionais e os usuários, desde a busca pela assistência até o diagnóstico e o tratamento.
Рага avaliação dessa dimensão foram desenvolvidas 11 proposições e, para melhor visualização, os resultados foram agrupados nos itens técnico-científico, interpessoal e ambiental. 
Tabela 3: Percentual das respostas referentes à proposições para avaliação da dimensão processo. Município da Região Metropolitana de Campinas, SP, 2008

\begin{tabular}{|c|c|c|c|c|c|c|}
\hline & \multirow{2}{*}{ Proposições } & \multicolumn{5}{|c|}{ Escala de Likert } \\
\hline & & 1 & 2 & 3 & 4 & 5 \\
\hline \multirow{3}{*}{$\begin{array}{l}\text { TÉCNICO- } \\
\text { CIENTÍFICO }\end{array}$} & $\begin{array}{c}\text { A equipe de enfermagem que me atendeu prestava os } \\
\text { cuidados com segurança. }\end{array}$ & 2,3 & 2,3 & 3,8 & 20,4 & 71,2 \\
\hline & $\begin{array}{c}\text { Os procedimentos eram realizados corretamente pela equipe } \\
\text { de enfermagem. }\end{array}$ & 4,2 & 1,2 & 3,8 & 16,2 & 74,6 \\
\hline & $\begin{array}{l}\text { O profissional da enfermagem não utilizou luvas ao prestar os } \\
\text { cuidados }\end{array}$ & 76,2 & 3,1 & 3,8 & 8,5 & 8,5 \\
\hline \multirow{5}{*}{ INTERPESSOAL } & Fui tratado com respeito pela equipe de enfermagem & 0,4 & 0,4 & 2,3 & 16,9 & 80 \\
\hline & $\begin{array}{l}\text { Os profissionais da enfermagem que me atenderam } \\
\text { chamavam-me pelo nome. }\end{array}$ & 3,1 & 1,9 & 8,5 & 9,6 & 76,9 \\
\hline & $\begin{array}{c}\text { Os profissionais de enfermagem não se apresentavam antes } \\
\text { de prestar os cuidados. }\end{array}$ & 65,8 & 4,2 & 10 & 6,9 & 13,1 \\
\hline & $\begin{array}{l}\text { A equipe de enfermagem ajudou-me a compreender o meu } \\
\text { estado de saúde. }\end{array}$ & 9,6 & 1,5 & 10,8 & 25,8 & 52,3 \\
\hline & $\begin{array}{c}\text { A enfermagem foi clara ao informar sobre meu problema de } \\
\text { saúde. }\end{array}$ & 5,8 & 2,3 & 9,6 & 27,3 & 54,6 \\
\hline \multirow{4}{*}{ AMBIENTAL } & $\begin{array}{c}\text { Esperei muito tempo pelo atendimento de enfermagem } \\
\text { quando cheguei à unidade. }\end{array}$ & 46,5 & 10,8 & 2,3 & 21,2 & 19,2 \\
\hline & A equipe de enfermagem realizava os exames prontamente & 9,2 & 5 & 10,8 & 24,6 & 50,4 \\
\hline & $\begin{array}{l}\text { Percebi que os usuários em situação grave eram atendidos } \\
\text { com rapidez. }\end{array}$ & 6,9 & 1,5 & 3,8 & 16,9 & 70,8 \\
\hline & $\begin{array}{c}\text { Os profissionais da enfermagem que me atenderam } \\
\text { chamavam-me pelo nome. }\end{array}$ & 3,1 & 1,9 & 8,5 & 9,6 & 76,9 \\
\hline
\end{tabular}

\section{Dimensão de Resultado}

O resultado é a obtenção das características desejáveis dos produtos ou serviços, retratando os efeitos da assistência na saúde do usuário e da população.

Considerando que tais efeitos estão intimamente relacionados com as expectativas dos usuários em relação ao serviço, as proposições avaliativas foram agrupadas em performance dos serviços, que indica se a oferta promoveu o que o usuário necessitava, esperava ou desejava; a equidade, que enfoca o julgamento de justiça distributiva, processual e interacional em relação ao tratamento recebido por outros usuários e o acolhimento, que envolve as emoções que os usuários experimentaram na obtenção dos resultados da assistência recebida.

Nesta investigação, procurou-se avaliar a dimensão resultado por meio de 11 proposições. 
Tabela 4: Percentual das respostas referentes à proposições para avaliação da dimensão resultado. Município da Região Metropolitana de Campinas, SP, 2008.

\begin{tabular}{|c|c|c|c|c|c|c|}
\hline & \multirow{2}{*}{ Proposições } & \multicolumn{5}{|c|}{ Escala de Likert } \\
\hline & & 1 & 2 & 3 & 4 & 5 \\
\hline \multirow{5}{*}{ PERFORMANCE } & $\begin{array}{l}\text { Observei melhora de minha saúde com os cuidados } \\
\text { realizados pela equipe de enfermagem. }\end{array}$ & 3,1 & 1,5 & 6,9 & 23,5 & 65 \\
\hline & $\begin{array}{l}\text { O tratamento que recebi pela equipe de enfermagem } \\
\text { possibilitou uma rápida melhora do meu estado de saúde }\end{array}$ & 3,1 & 1,2 & 4,6 & 22,3 & 68,8 \\
\hline & $\begin{array}{l}\text { A equipe de enfermagem cometeu erros ao prestar os } \\
\text { cuidados }\end{array}$ & 84,2 & 2,7 & 3,5 & 2,3 & 7,3 \\
\hline & $\begin{array}{c}\text { A enfermagem explicou os critérios de classificação por cores } \\
\text { para o atendimento na unidade }\end{array}$ & 15,8 & 1,5 & 11,2 & 26,2 & 45,4 \\
\hline & $\begin{array}{c}\text { A equipe de enfermagem não explicou os procedimentos que } \\
\text { realizou. }\end{array}$ & 14,2 & 9,6 & 5,0 & 5,4 & 65,8 \\
\hline \multirow{3}{*}{ EQUIDADE } & $\begin{array}{l}\text { A equipe de enfermagem não explicou os motivos pelos } \\
\text { quais não podia atender-me de imediato. }\end{array}$ & 10 & 5,8 & 14,2 & 23,5 & 46,5 \\
\hline & $\begin{array}{l}\text { Não fui informado sobre os serviços existentes que poderiam } \\
\text { dar continuidade ao meu atendimento }\end{array}$ & 29,2 & 5 & 46,5 & 8,8 & 10,4 \\
\hline & $\begin{array}{c}\text { Os cuidados prestados pela equipe de enfermagem foram } \\
\text { humanizados. }\end{array}$ & 0,8 & 0.4 & 3,5 & 20 & 75,4 \\
\hline \multirow{3}{*}{ ACOLHIMENTO } & $\begin{array}{l}\text { A equipe de enfermagem considerou minha opinião durante } \\
\text { o tratamento }\end{array}$ & 8,1 & 3,5 & 11,5 & 25,4 & 51,9 \\
\hline & $\begin{array}{l}\text { Em nenhum momento fui questionado se estava satisfeito } \\
\text { com o atendimento }\end{array}$ & 48,8 & 4,2 & 13,8 & 15,8 & 17,3 \\
\hline & $\begin{array}{l}\text { Não permitiram que meu acompanhante permanecesse ao } \\
\text { meu lado }\end{array}$ & 24,2 & 9,6 & 36,5 & 4,6 & 25 \\
\hline
\end{tabular}

\section{Escore de favorabilidade}

A análise do escore de favorabilidade demonstrou a percepção dos usuários perante o objeto do estudo. Quanto maior o escore obtido, mais favorável é a atitude/percepção do sujeito e vice-versa.

Por esta avaliação, a dimensão de resultado obteve o melhor escore de favorabilidade, com média de 39,84 (dp 土 4,66), mínimo de 22,00 e máximo de 51,00, com diferença significante, $p<0,001$. A dimensão de estrutura, por sua vez, apresentou o pior escore de favorabilidade, média de 36,48 (dp \pm 4,23), mínimo de 24,00 e máximo de 49,00, quando comparada às dimensões de processo e de resultado, apresentando diferença estatisticamente significante $p<0,001$.

No que tange ao motivo de procura pela unidade em relação ao domínio estrutura, constatou-se divergência significativa, pois o grupo não urgência difere significativamente do grupo urgência, apresentando valor maior. Portanto, os usuários não urgentes apresentaram maior insatisfação com a estrutura da UEA.

\section{DISCUSSÃO}

Para caracterizar o motivo do atendimento na UEA foi adotada a divisão de atendimento que decompõe a complexidade assistencial dos usuários em cinco divisões, a saber: ressuscitação - situações de risco à vida ou com sinais de deterioração do quadro clínico; emergência condições que potencialmente ameaçam a vida ou requerem rápida intervenção; urgência - condições que podem progredir para um problema sério; semi-urgência - condições que apresentam potencial para complicações ou relacionadas à idade do paciente; não urgência condições agudas não urgentes, ou problemas crônicos sem sinais de deteriorização(9).

Assim, verificou-se que os usuários classificados em semi-urgências e não urgências representaram 168 (64,6\%) dos usuários, enquanto 92 (35,3\%) foram classificados como urgência e emergência, refletindo na maior ocupação do setor azul (pronto atendimento), caracterizado como o setor com o maior número de usuários 129 (49,6\%).

A superlotação das portas hospitalares de urgência no que se refere a usuários com necessidades de cuidados de baixa complexidade é decorrente de vários fatores, dentre os quais se destacam a baixa resolutividade da 
rede primária, a desarticulação entre os níveis de assistência e a própria insuficiência estrutural, gerencial e funcional desses serviços ${ }^{(10)}$.

No que tange à dimensão de estrutura observou-se pela Tabela 2, que a maioria dos sujeitos mostrou percepção favorável referente à qualidade e organização na estrutura da UEA no que se refere a: conforto, equipamentos, limpeza dos sanitários, acesso a unidade, sinalização interna e medicamentos.

Verificou-se, também, que $55,4 \%$ dos usuários discordaram que o estado de conservação da unidade era precário, no entanto, uma parcela considerável de participantes, $35 \%$, concordou com a precariedade da unidade.

O ambiente exerce forte influência sobre os indivíduos e pode estimular ou inibir a interação entre os envolvidos. Engloba não só o espaço físico utilizado pelas pessoas, como também as próprias pessoas que interagem nesse contexto, a sua cultura, o mobiliário, o arejamento, a temperatura, os ruídos e as condições de espaço(11).

Referente ao respeito à privacidade do usuário na unidade de emergência, não houve clareza dos respondentes. Segundo a Carta dos Direitos dos Usuários da Saúde, deve ser assegurado ao cidadão, o atendimento acolhedor e livre de discriminação, visando à igualdade de tratamento e uma relação pessoal e saudável, destacando-se o direito à privacidade nas consultas, procedimentos diagnósticos, preventivos, cirúrgicos, terapêuticos e internações. A privacidade também diz respeito à proteção da intimidade do usuário, que muitas vezes pode ser garantida com uso de divisórias ou até mesmo com cortinas e elementos móveis que permitam ao mesmo tempo integração e privacidade, facilitando o processo de trabalho, aumentando a interação da equipe e ao mesmo tempo possibilitando atendimento personalizado ${ }^{(12)}$.

Com relação à capacitação e quantitativo de profissionais de enfermagem, averiguou-se que os maiores percentuais concentraram-se no grau de concordância total.

Desenvolver pessoas e melhorar a qualidade da assistência à saúde beneficia tanto os trabalhadores como os pacientes, considerando seus direitos como cidadãos contribuindo para melhorar a qualidade de vida das pessoas ${ }^{(13)}$.
A análise da dimensão de processo mostrou por meio da Tabela 3 que os itens que obtiveram melhores percentuais foram: o respeito dispensado pela equipe de enfermagem (96,9\%); a segurança na realização dos cuidados (91,6\%); a realização dos procedimentos corretamente (90,8\%); a rapidez no atendimento aos usuários em estado grave (87,7\%); os profissionais de enfermagem chamar os usuários pelo nome $(86,5 \%)$ e a clareza com que os usuários eram informados quanto ao seu estado de saúde pela equipe de enfermagem (82,3\%).

Notou-se, também, que do total de participantes, $79,3 \%$ discordaram que o profissional de enfermagem não utilizou luvas para prestar o atendimento.

Cabe salientar que as luvas compõem o arsenal do Equipamento de Proteção Individual (EPI), padronizadas nas medidas de Precauções Universais e tem a finalidade primordial de proteger os profissionais de saúde da exposição ao sangue ou a outros fluidos corporais, como secreções e excretas. Assim, reduz-se o contato direto das mãos do profissional com tecidos do usuário, lesões, membranas, mucosas, por exemplo, na realização de procedimentos invasivos ${ }^{(14)}$. Nesse sentido, condutas como essa necessitam estar permanentemente sob processo de avaliação, pois sabe-se que as intervenções técnicas, realizadas predominantemente pelo pessoal técnico junto à clientela, comportam riscos e podem comprometer a qualidade do cuidado ofertado.

Quanto à falta de apresentação do profissional de enfermagem antes de prestar os cuidados ao usuário, $70 \%$ discordaram dessa afirmativa e, no que diz respeito à ajuda fornecida pela equipe de enfermagem na compreensão do usuário sobre seu estado de saúde, $78,1 \%$ assinalou concordância em relação a essa proposição.

Os usuários têm o direito de possuir profissionais que se responsabilizem por seu atendimento e que esses profissionais sejam facilmente identificados por meio de crachás visíveis ou por outras formas de identificação de fácil percepção. Possui, também, o direito de ter o tratamento adequado e efetivo para seu problema, visando à melhoria da qualidade dos serviços prestados, tendo garantido informações sobre o seu estado de saúde, extensivas aos seus familiares e/ou acompanhantes, de maneira clara, objetiva, respeitosa, compreensível e adequada à condição cultural, 
respeitando os limites éticos por parte da equipe de saúde ${ }^{(12)}$.

Não houve clareza dos respondentes na proposição que considerava que o usuário esperou muito tempo para 0 atendimento de enfermagem.

Tal resultado aproxima-se daqueles encontrados na pesquisa nacional: "A Saúde na Opinião dos Brasileiros", que aponta que o atendimento prestado por equipes de enfermagem em estabelecimentos SUS é, de modo geral, muito bem avaliado. Um único item com avaliação negativa refere-se ao tempo de espera para o atendimento(1).

A dimensão de resultado revelou, conforme Tabela 4, que houve elevada concordância nas questões que tratavam da humanização nos cuidados $(85,4 \%)$, melhoria da saúde com os cuidados realizados (88,5\%) e sobre a rápida melhora da saúde com o tratamento executado pela equipe de enfermagem $(91,1 \%)$.

O cuidado de enfermagem é de primordial importância para a hospitalização, uma vez que é o que permite estabelecer intervenções terapêuticas seguras aos usuários ${ }^{(15)}$.

Essa segurança pode ser considerada como critério de satisfação, pois na prática cotidiana são reconhecidas circunstâncias que relacionam os eventos adversos e que culminam em erros para a atenção à saúde ${ }^{(16)}$.

Verificou-se que $33 \%$ dos usuários concordaram, sendo $15,8 \%$ parcialmente e $17,3 \%$ totalmente, com a afirmativa de não terem em nenhum momento sido questionados se estavam satisfeitos com o atendimento.

Ressalta-se a importância do atendimento das expectativas dos usuários dos serviços de saúde, haja vista que a satisfação do usuário subsidiará a tomada de decisão para a melhoria da assistência nas referidas instituições ${ }^{(17)}$.

As questões referentes à consideração da opinião do usuário sobre o seu tratamento e se foi explicado o critério de classificação por cores para o atendimento, obtiveram percentual de concordância maior que $70 \%$.

A importância da satisfação dos usuários com esse quesito repousa sobre a crescente demanda e procura dos serviços de urgência e emergência, onde é possível observar um enorme fluxo de circulação desordenada dos usuários nas portas das UEA, tornando-se necessária a reorganização do processo de trabalho desses serviços, de forma a atender os diferentes graus de especificidade e resolutividade na assistência realizada.

Em relação às afirmativas negativas referentes à explicação da equipe de enfermagem quanto ao não atendimento imediato e aos procedimentos que realizou, o percentual de discordância apresentou-se elevado e não houve clareza dos respondentes sobre a permanência de acompanhante durante o período de atendimento.

A Carta dos Direitos dos Usuários da Saúde garante o direito ao acompanhamento por pessoa de sua livre escolha nas consultas, exames e internações, no momento do pré-parto, parto e pós-parto e em todas as situações previstas em lei (criança, adolescente, pessoas vivendo com deficiência ou idosos). Nas demais situações, o direito a acompanhante e/ou visita diária, reserva-se a um período não inferior a duas horas durante as internações, ressalvadas as situações técnicas não indicadas ${ }^{(12,18)}$.

Em virtude do que foi mencionado, pode-se inferir que a dimensão de estrutura requer maior atenção por parte dos gerentes da instituição pesquisada, uma vez que foi a que os acompanhantes-usuários mais pontuaram deficiências.

Decorrente das especificidades do processo de produção em saúde, nem uma estrutura adequada garante bons resultados e nem uma estrutura ruim os impede; processos desenvolvidos com rigor da técnica podem ou não atingir os resultados com excelência. Assim, as atuações estão sempre nos limites das possibilidades, onde uma estrutura adequada favorece processos adequados, que por sua vez aumenta a probabilidade do alcance de resultados desejáveis ${ }^{(19)}$.

Frente aos resultados apresentados e como as três dimensões estão intimamente interligadas e são interdependentes, torna-se imperativo o monitoramento das mesmas, a fim de que repercussões indesejáveis à qualidade assistencial sejam evitadas.

\section{CONCLUSÃO}

A realização desta investigação atingiu os seus objetivos na medida em que possibilitou a avaliação da qualidade assistencial de uma UEA na perspectiva de seus usuários. Os achados permitiram, ainda, verificar que a dimensão que apresentou pior escore foi a de dimensão de estrutura, sugerindo que os usuários tendem a considerar os aspectos referentes à estrutura como os 
deficitários para a assistência e que a dimensão de resultado obteve o melhor escore de favorabilidade.

Constatou-se, portanto, que pesquisar a satisfação dos usuários dessa unidade contribuiu para a gestão interna, uma vez que proporcionou o conhecimento das necessidades e expectativas desses usuários, indicando estratégias que influenciam no nível de qualidade dos serviços prestados.

\section{REFERÊNCIAS}

1. Conselho Nacional de Secretários de Saúde. A saúde na opinião dos brasileiros. Brasília; 2003.

2. Motta FCP. Teoria geral da administração: uma introdução. São Paulo: Pioneira; 2002.

3. Corrêa HL, Caon M. Gestão de serviços: lucratividade por meio de operações e satisfação dos clientes. São Paulo: Atlas; 2002.

4. Turrini RNT. Lebrão ML, César CLG. Resolutividade dos serviços de saúde por inquérito domiciliar: percepção do usuário. Cad. Saúde Pública, 2008;24(3):663-74.

5. Donabedian A. Evaluating the quality of medical care. Milbank Mem Fund Q. 1966;44(3):166-203.

6. Donabedian A. La calidad de la atención médica- definición y métodos de avaluacíon. Mexico: La Prensa Mexicana; 1984. 7. Donabedian A. The methods and findings of quality assessment and monitoring: an illustraded analysis. Michigan: Health Administration Press; 1985.

8. Donabedian A. The quality of medical care: how can it be assessed? JAMA. 1988; 260(12):1743-8.

9. Pires PS. Escala Canadense de Triagem e acuidade (CTAS): validação e aplicação. Revista Emergência. 2005;1(2):14-9. 10. Barros AF, Walker G, Pessati AS. Critérios para organização dos hospitais QualiSus. Brasília: Ministério da Saúde; 2004. 11. Stefanelli MC. Conceitos teóricos sobre comunicação. In: Stefanelli MC, Carvalho EC, organizadoras. A comunicação nos diferentes contextos da enfermagem. $2^{\mathrm{a}}$ Ed. Barueri: Manole; 2012.

12. Ministério da Saúde. Carta dos Direitos dos Usuários da Saúde. Brasília; 2011.

13. Nepomuceno LMR, Kurcgant P. Uso de indicador de qualidade para fundamentar programa de capacitação de profissionais de enfermagem. Rev. esc. enferm. USP. 2008;42(4):665-72.

14. Centers for Disease Control and Prevention. Guidelines on Hand Hygiene in Health Care [Internet]. 2009. [cited 2012 Set 26]. Available from:

http://www.cdc.gov/mmwr/PDF/rr/rr5116.pdf

15. Machado WC. O trabalho no setor saúde. In: Geovanini T, Moreira A, Schoelle DS, Machado WC. História da enfermagem: versões e interpretações. $2^{a}$ ed. Rio de Janeiro: Revinter; 2009. 16. Wegner W, Pedro ENR. A segurança do paciente nas circunstâncias de cuidado: prevenção de eventos adversos na hospitalização infantil. Rev Lat Am Enferm. 2012; 20(3):[8 telas]. 17. Pena MM, Melleiro MM. Grau de satisfação de usuários de um hospital privado. Acta paul. enferm. [Internet]. 2012 [cited 2012 Set 26]; 25(2):197-203. Available from: http://www.scielo.br/pdf/ape/v25n2/en_a07v25n2.pdf 18. Vieira GB, Alvarez AM, Girondi JB. O estresse do familiar acompanhante de idosos dependentes no processo de hospitalização. Rev. Eletr. Enf. [Internet]. 2011 [acesso em: 29 mar 2013];13(1):78-89. Disponível em:

http://dx.doi.org/10.5216/ree.v13i1.8719.
Desse modo, acredita-se que essa avaliação possa direcionar ou redirecionar as atividades desenvolvidas, permitindo o monitoramento dos resultados, a identificação de desvios e subsidie o planejamento e a organização de ações para a resolução de eventuais problemas.

19. Malik AM. Qualidade e avaliação nos serviços de saúde: uma introdução. In: D'Innocenzo M, coordenador. Indicadores, auditorias, certificações: ferramentas de qualidade para gestão em saúde. São Paulo: Martinari; 2006.

Artigo recebido em 05/08/2011. Aprovado para publicação em 04/06/2012. Artigo publicado em 31/03/2013. 